\title{
LAS CONSTRUCCIONES PASIVAS EN EL ESPAÑOL DEL SIGLO XV
}

\author{
AMPARO RICOS VIDAL \\ (Universidad de Valencia)
}

1. Las construcciones que recogen el sentido pasivo en el siglo XV son: ser + participio y ser + forma verbal'. Estas mismas estructuras poseen, en esta época, significados distintos debido a una serie de fenómenos que confluyeron en el bajo latín y que fueron diferenciándose durante los primeros siglos de la lengua.

El latín presentaba una conjugación heterogénea con formas sintéticas en el tema de infectum y perifrásticas en el de perfectum. La evolución fonética y el empuje de estas últimas, que ya en latín vulgar adquirieron valor de presente, dio lugar a la creación de un sistema homogéneo formado por perífrasis del verbo esse y el participio en -tus, sistema que recogieron las lenguas romances (SASSOLS: 1948, 148-150; JORDAN-MONOLIU: 1972, 334; HARRIS: 1978, 189; LAROCHETTE: 1939, 396; ERNOUT: 1964, 228-9).

Al mismo tiempo los verbos intransitivos, a semejanza de los reflejos, crearon un participio y pasaron a utilizar esta misma forma como perfecto (HANSSEN: 1945, 230232; BASSOLS: 1948, 195; KUKENHEIM: 1968, 65; PAR: 1898/1923: 312).

De esta manera, la construcción pasiva recogía, en este momento, tres valores: el de presente, el de perfecto y el de estado resultante del proceso.

Durante la época medieval, todos estos valores van distinguiéndose gracias la uso de dos nuevos auxiliares: estar y aver que se generalizaron bien entrado el siglo XIV, manteniéndose las confusiones antes mencionadas aún en el siglo XV.

Pero junto con la forma ser + participio, el español presenta, desde sus inicios, una estructura pasiva alternativa: se + forma verbal (LAPESA: 1942/83,216). Esta construcción adquirió en el período romance valor pasivo, hasta convertirse en la actualidad, en la forma más común de expresar estos contenidos (MONGE: 1955, 90-91).

'El problema de la "voz pasiva" en español ha sido uno de los lcmas más debatidos desde que ALARCOS LLORACH (1951/80, 1965/80) escribió sus artículos en los que, desde un punto de vista funcional negaba lá existencia en español de una estructura gramalical determinada que recogiera los contenidos pasivos.

Esta perspectiva dio lugar a una amplia polémica a la que se han aportado menos argumentos tanto en favor (CESAR HERNANDEZ: 1984) como en contra (MANACORDA DE ROSETTI: 1961/79), CARRASC(): 1973, LAZARO CARRETER: 1980) de la pasiva. 
2. Los resultados del análisis de varias obras del siglo $X^{2}$ nos muestran el predominio de la forma perifrástica sobre la refleja.

Esta superioridad numérica de ser + participio, que sorprende ante el escaso empleo de dicha construcción en español actual, nos hace pensar en un uso estilístico de la misma en la época que describimos. Nos centraremos, por tanto, en este artículo, en aquellos valores que permitan demostrar nuestra hipótesis.

3. La forma con participio recoge confundidos, aún en este período, los tres valores a los que antes hacíamos referencia:

a) Valor de infectum latino (formas simples):

En ejemplos como:

"Est nos es denegada

de Mares la tal vitoria" (P. Marqués,192,513-514)

"...Por todos los Reynos christianos y otras regiones era creido el contrario." (C. Reyes, 73,15)

b) Valor del perfectum latino (formas compuestas):

Aunque aparecen casos con aver documentados ya en el siglo XIV, hemos de decir que encontramos con frecuencia formas simples con significación perfecta en los textos del XV ( 85 casos frente a 16 con aver):

"Su muger de Admeto, rey de Tesalia, que sabicndo que era proficlizado por cl dios Apolo que su marido recebería muerte si no uviese quien voluntariamente la tomase por él, con alegré voluntad, porque el rey biviesé, dispuso de se malar." (Cárcel, 131, 3-8)

c/Valor de estado resultante de la acción anterior:

En muchas ocasiones, el contexto no precisa cuál es el verdadero valor de la construcción y se mantiene la ambigüedad. A esto se añade que la diferencia entre un perfecto pasivo y el estado resultante de la acción anterior, depende del enfoque con que se mire la construcción, puesto que la acción acabada, perfecta que expresa el perfecto puede considerarse un estado.

Hallamos ejemplos en que no es posible discernir si se trata de la expresión de la acción perfecta o del estado resultante:

"Asimesmo, despues este rey don Enrrique se fue a ver con el rey don Luys de Franç̧ia a las vistas de Fuenterrabia, que en su corónica más por estenso son escriptas." (C.Reyes, 50 ,29-32)

Tales confusiones desaparecen en el momento en que la lengua desarrolla el empleo de los otros dos auxiliares (estar y aver), descargando al verbo ser de su carga funcional, por lo que se restringe su uso cada vez más, pasando a actuar como verbo copulativo y auxiliar de pasiva en la construcción con participio.

Estar y haber se documentan desde los primeros textos románicos, pero será en el siglo XIV cuando ambos verbos vean aumentado su uso, sobre todo el segundo, en las formas compuestas de los verbos (YLLERA: 1980).

4. De gran interés es el estudio de los casos en los que se expresa el Complemento

${ }^{2}$ El vaciado de los libros cilados en el apéndice bibliográfico nos proporcionó 2775 ejemplos de estas construcciones, de los cuales 1837 presentaban ser + participio con valor pasivo y 515 la forma refleja. 
Agente (C. AG.), ya que este confiere a la estructura un valor estilístico.

4.1.Como en la actualidad, el C.AG. no aparece necesariamente en la oración pasiva. En muchas ocasiones (desconocimiento, deseo de ocultarlo, modestia, respeto,...), este se evita, resaltando de esta manera, la acción verbal que recae sobre el O.D. de la activa, ahora sujeto gramatical.

Así en nuestro corpus no es habitual explicitar el agente de la acción (sólo un 18'6\% de media de los ejemplos lo llevan).

Sin embargo, destaca una serie de oraciones en las que la forma posiva es frecuente cuando se introduce en el diálogo la primera y segunda persona como agente:

"TTú scrás muerta sin justicia y de mí llorada sin razón!" (Cárcel, 101, 26) "Que después que me mudé al otro barrio, no han sido de mí visil adas" (Celest., 111,24) "Te ruega mi señora séa de lí visitada" (Celest., 195, 25) "para que tan presto me hubieses de pagar en la misma moneda el beneficio que por tí me fue demandado" (Celest. 197, 24) "En les (epilafios) que pudieron por mí ser leidos" (Lab. 127, 1229) "El que non quisiere poner diligençia,/por mí non puede ser más esperado" (P. Crítica, 45, 63-64)

"En la su corte es ya tanta maliçia,/ aquí non podía por mí ser contada." (P. Crítica, 1(2, 3-4)

Parece indicado creer que este tipo de construcción no se beve a un intento por parte del autor de hacer recaer la atención del lector u oyente sobre el "sujeto paciente", ya que en ese caso el C. AG. no iría expresado. Si a esto añadimos que debido a la flexión clara y reconocible del verbo español no es preciso expresar el sujeto agente cuando este es de primera o segunda persona, a no ser que queramos enfatizarlo (GILI GAYA: 1961/85, 25), podemos pensar que el papel de dicho complemento en los ejemplos citados, no es el de mostrar, simplemente, el agente de la acción, que vemos indicado en los morfemas verbales, sino realzar, todavía más, la función agentiva que en la acción activa hubiera quedado relegado. agente $^{3}$.

Entendemos este hecho como un medio expresivo de enfatización o tematización del

4.2. Ya en el Cid (MENENDEZ PIDAL: 1908/54, 343), la preposición de predomina como introductora del régimen pasivo en los textos castellanos, al igual que ocurre en nuestro corpus (de 339 casos con C. AG. explicito, 220 son introducidos por de y 119 por por).

El test de Pearson aplicado a nuestros resultados, nos señala que la repartición de ambas preposiciones no se debe al azar ${ }^{4}$. Por lo que pensamos que corresponde a un deseo específico del autor (o autores) de inferir a la construcción un valor estilístico.

En este sentido, Keniston (1937, 472-3) indica que, aunque no está totalmente fijado el uso de estas preposiciones, la tendencia general, en el siglo XVI, es utilizar de cuando la acción es indeterminada (universal, durativa o iterativa) y cuando el agente está también indeterminado, y por cuando la acción es determinada (perfecta) y el agente también lo está.

Esta misma tendencia que observa Keniston en el siglo XVI, se percibe en los textos del XV. Sin embargo, no se puede hablar de una generalización de estos valores, puesto

${ }^{3}$ Creemos que este hecho está relacionado con la importancia que en español tienc la categoría persona, por lo que nos proponemos estudiar en el futuro las posibles relaciones entre dicha categoría y la vo\% pasiva.

4 La aplicación del lest de Pearson a nuestros ejemplos nos da el resultado de 0'10-0’05, to que significa que tenemos de 5 a 10 probabilidaes sobre 100 de equivocarnos rechazando la hipólesis nula, es decir, aceptando que no se trata de una repartición al arar. 
que aunque por aparece siempre con un agente determinado, de recoge a su vez este valor introduciendo tanto agentes determinados como indeterminados.

No obstante, y como señala Keniston, la mayoría de los casos con de se refieren a indeterminados:

\section{CON DE:}

"Los reyes crueles de todos los ombres son desamados"(Cárcel, 104, 20)

Con agente determinado:

"este almirante fué vencido del maestro de Santiago su enemigo" (C. Varones, 24,5-

6)

CON POR:

"E la tal graçia graciosa

por Dios a vos otorgada." (P. Marqués,75, 22-23)

5. Descubrimos en nuestros ejemplos otros empleos de la perífrasis con participio que apoyan la hipótesis del uso estilístico de dicha construcción.

El orden de la perífrasis imperante es el actual, con el verbo auxiliar antepuesto al participio. Ahora bien, hay una serie de ejemplos en los que se mantiene el orden latino (participio + ser).

En general, se trata de situaciones en las que se enfatiza el valor léxico del pàrticipio. Así lo encontramos en:

-ORACIONES CONDICIONALES:

"Y si sentidos fuésemos, a ti y a mí librarían de toda la gente de tu padre." (Celest. $225,16-17)$

-PRECEDIDO DEL ADVERBIO TAN O CUAN:

"dixo que nunca tan obedeçido fuera de las gentes, ni tan bien aguardado." (Vict. 16, 29-30)

-EN EXPRESIONES EXCLAMATIVAS:

"ILoado sea Dios que me quiso tanto mal!" (Cor. 156,2)

-EN POSICION INICIAL TRAS PAUSA:

"Lastimado serás brevemente con la muerte de tu única hija." (Celest. 290,12)

En interior de oración tras pausa es corriente la anteposición del auxiliar, pero tras pausa inicial absoluta encontramos sólo 8 casos de este esquema, todos en fragmentos narrativos, siendo más general la posposición.

\section{-CALCOS LATINOS:}

"Segund dicho es" (Vict. 281,10)

-OTROS CASOS (JUEGOS DE PALABRAS, ANTEPOSICION DEL AGENTE,...):

"Pues mucho deviere ser más que culpado/ aquel coraçón que si non querer/ quicre, que quicre querido) non ser,/ o por ser querido bive despagado" (Lab. 108,893-6). "E mas le digo que la más sutil muger de estado, que del rey amada sea..." (Cor. 81,20)

Si tenemos en cuenta todo esto, podemos catalogar la posposición del verbo ser como un rasgo prosódico gracias al cual se enfatiza el elemento portador del significado: el participio.

6. En español medieval, al igual que en otras lenguas como el italiano o el francés 
actual, los verbos intransitivos, los reflexivos y los que proceden de antiguos deponentes forman el perfecto con el auxilio de ser.

En español, la pugna entre ser y aver en estos casos, se inició muy pronto. En el Cid, se hallan ya ejemplos de este último verbo. En el siglo XIII fue usual el empleo de ser. Es la época de auge de esta construcción que vio decrecido su uso en el siglo XIV, a la vez que triunfaba la tendencia contraria (YLLERA: 1980,234).

En el siglo XV, nuestros textos recogen el predominio de ser frente a aver, lo que muestra un retroceso en el avance que este segundo auxiliar realizaba con este tipo de verbos.

-Verbos intransitivos:

"Llégate presto Sosia, que el triste de nuestro amo es caído del escala y no habla ni

se bulle." (Celest. 284,8-9)

- Verbos reflejos:

"Cuando Bruto supo cómo Dorotea venía fue muy maravillado." (Vict. 174,24) -Antiguos deponentes:

"Pues cuantas hoy son nacidas, que de ella tengan noticia, se maldicen, querellan a Dios." (Celest. 150,1).

A este respecto Yllera $(1980,234)$ señala que las formas con aver son más frecuentes en la Embajada a Tamorlán del madrileño Ruy González de Clavijo, escrita en los primeros años del siglo XV.

"Esto deja suponer que en la lengua familiar aver había triunfado ya con estos verbos a principios del XV, puesto que así lo alestigua una obra de escasas prelensiones literarias como la Embajada a Tamorlán. Por el contrario, el empleo de ser, que comenzaba a ser arcaizante, se consideraba más culto, de ahí su neto predominio en el lenguajc cuidado." (YLLERA: 198(), 234)

Este fenómeno que indica la autora apoya nuestros argumentos.

7. Basándonos en los datos aportados, creemos que se puede hablar de un empleo estilístico de ser + participio en el español del siglo XV.

Todos los gramáticos del español actual son unánimes a la hora de señalar que el español prefiere la construcción activa a la pasiva, y que la forma con ser ha visto disminuido su empleo frente al aumento creciente de la pasiva refleja (ALCINA-BLECUA: 1975/83, HANSSEN: 1945, 245, GILI GAYA: 1961/85, 122, ESBOZO: 1973/86, 379).

No obstante, vemos como en el siglo XV el uso de ser + participio predomina sobre el de la pasiva refleja. Para algunos autores (ALCINA-BLECUA: 1975/83, 903) se debe al espíritu humanista y culto de este período que intenta ennoblecer la lengua española introduciendo el mayor número posible de latinismos (tanto léxicos como sintácticos).

La construcción pasiva perifrástica es una estructura sintáctica que hace posible un acercamiento a la lengua latina, ya que en esta lengua se encuentra el verbo esse y el participio en -tus con distintos valores. A la vez posibilita, al estar formada por varios elementos, el juego de palabras y el hipérbaton que caracteriza, no sólo a determinados autores más latinizantes (Juan de Mena o Diego de San Pedro), sino a todos ellos en general, puesto que estos juegos con el auxiliar y el participio los encontramos en escritores que plasman, aunque sea parcialmente, el lenguaje vulgar de la época (Arcipreste de Talavera o Fernando de Rojas).

Por tanto, podemos deducir que el uso de ser + participio se consideraba, en el siglo 
$\mathrm{XV}$, más culto, por lo que predomina en el lenguaje escrito.

De ahí que se emplee, no sólo en los juegos de palabras o en aquellas situaciones en que se enfatiza el elemento portador del significado de la perífrasis, sino también, en construcciones especiales que realzan el agente de la acción, a la vez que se distribuye, y no de una manera fortuita, las funciones de las preposiciones que generalmente acompañan a este (de y por).

A nuestro entender, y en esto nos unimos a la opinión de A. Yllera, se debe también a esta preferencia el que perviva la forma con participio como perfecto de los verbos intransitivos y reflexivos, siendo que las formas compuestas con aver, para este caso, se encontraban ya ampliamente documentadas en el siglo anterior.

El uso de la pasiva refleja, al igual que el perfecto de verbos intransitivos con aver, debió de ser la forma más utilizada en el lenguaje popular, ya que el español, a diferencia de otras lenguas románicas, no ha conservado estas formas con participio, y el empleo de la pasiva con se ha desbancado a la construcción perifrástica adquiriendo nuevos valores en español que son estadios de un proceso que lleva a la conversión del pronombre se en el índice de la impersonalidad en español actual.

\section{Referencias bibliográficas}

\section{TEXTOS LITERARIOS}

Cárcel: SAN PEDRO, Diego (1493): Cárcel de amor. Ed. de E. Moreno Báez, Cátedra, Madrid, 1982.

Celest.: DE ROJAS, Fernando (Burgos 1499, Sevilla 1501): La Celestina. Ed. de Bruno María Damiani, Cátedra, Madrid, 1979.

Cor.: MARTINEZ DE TOLEDO, Alfonso (1466): Arcipreste de Talavera o Corbacho. Ed. de Michael Berli, Cátedra, Madrid, 1979.

C. Reyes: ANONIMO (finales del XV): Crónica incompleta de los Reyes Católicos (14591476). Prólogo y notas de Julio Puyol, Madrid, Tipografía de Archivos Olózaga, 1934.

C. Varones: DEL PULGAR, Fernando (1486): Claros Varones de Castilla. Ed., introducción y notas de Jesús Domínguez Bodena, Espasa-Calpe, Madrid, 1969.

Lab.: DE MENA, Juan (1444): Laberinto de Fortuna. Ed. de John G. Cummins, Cátedra, Madrid, 1979.

P. Marqués: MARQUES DE SANTILlANA (1444): Poesías completas. I. Serranillas, cantares y decires. Sonetos fechos al itálico modo. Ed. de Manuel Durán, Ed. Clásicos Castalia, 1982.

P. Crítica: VARIOS: Poesía crítica y satírica del s. XV. Ed. de Julio Rodríguez Puértolas, Clásicos Cástalia, Madrid, 1981.

Vict.: DIEZ GAMES, Gutierre (segunda mitad del XV): El Victorial. Crónica de Don Pero Niño, conde dé Buelna. Ed. y estudio por Juan de Mata Carriazo, Espasa-Calpe, Madrid, 1940.

\section{BIBLIOGRAFIA CONSULTADA}

Alarcos Llorach, E.: "La diátesis en español" (II ) en Estudios de gramática funcional del español, Gredos, Madrid, 1965-67/80, (pp. 90-94)

Alarcos Llorach, E.: "Pasividad y atribución" (VIII) en E.G.F.E., 1951/80, (pp.163-171)

Alcina, J.-Blecua, J.A.: Gramática española, Ariel, Barcelona, 1975/83. 
Bassols de Climent: Sintaxis histórica de la lengua latina, T.II. C.S.I.C., Barcelona, 1948. Carrasco, Félix:"Sobre el formante de la voz pasiva en español" en R.S.E.L., 3, 1973. Ernout, A.-Thomas F.: Syntaxe latine.Paris. Edition Kleincksieck.1964

Gili Gaya, S.: Curso superior de sintaxis española, Ed. Bibliograf, Barcelona, 1961/85. Hanssen, F.: Gramática histórica de la lengua castellana, Buenos Aires, Librería El Ateneo, 1945.

Harris, M.: The evolution of French syntax. A comparative approach, Longman, Londres, 1978.

Hernández, C.: "Transitividad e intransitividad" (IX) en Gramática funcional del español, Madrid, Gredos, 1984.

Jordan, I.-Monoliu, M.: Manual de lingüistica románica, I, Gredos, 1972.

Keniston: Syntax of castiliaun prose. The sixteenth century, Chicago, Univ. Press, Chicago, 1937.

Kukenheim: Grammaire historique de la langue française, Universitaire Pers, Leiden, 1963. Lapesa, R.: Historia de la lengua española, Gredos, Madrid, 1942/83

Larochette: "Les aspects verbaux en espagnol ancien", RlaRo, 1939 (327-421)

Lázaro Carreter, F.: "Sobre la pasiva en español" en Estudios de lingüistica, Crítica, Barcelona, 1980/81.

Manacorda De Rosetti, M.: Estudios de gramática estructural, Buenos Aires, Paidós, 1979. Menéndez Pidal, R.: Cantar de Mío Cid. Textos, gramática y vocabulario, Espasa-Calpe, Madrid, 1908/53.

Monge, F.: "Las frases pronominales de sentido impersonal en español", en A.F.A. VII, 1955 (pp. 7-102), Zaragoza.

Par, A.: Sintaxi catalana segons los escrits de Bernat Metge, Halle Verlag von May Niemeyer. $1893 / 1923$.

R.A.E.: Esbozo de una nueva gramática de la lengua española, Espasa-Calpe, Madrid, 197386.

Yllera, A.: Sintaxis histórica del verbo español: las perifrasis medievales, Univ. Zaragoza, 1980. 